\title{
Progression of familial and non-familial dilated cardiomyopathy: long term follow up
}

\author{
V V Michels, D J Driscoll, F A Miller, T M Olson, E J Atkinson, C L Olswold, D J Schaid
}

Heart 2003;89:757-761

\begin{abstract}
Background: It is unknown whether progression of familial idiopathic dilated cardiomyopathy differs from progression in the non-familial form. It has been suggested that familial disease indicates a worse prognosis, and that this should be considered when planning the timing of heart transplantation.

Objective: To compare five year survival or time to heart transplantation in an unselected series of patients with dilated cardiomyopathy who had been evaluated for familial $v$ non-familial disease through the echocardiographic investigation of first degree relatives.

Design: Medical records were reviewed and questionnaires were mailed to all patients who had previously participated in a family based study of dilated cardiomyopathy. Information was gathered about survival, heart transplantation, and left ventricular ejection fraction (LVEF) measurements. Survival data were censored at the time of cardiac transplantation.

Results: Follow up data were obtained for 99 of 101 patients (69 with non-familial and 30 with familial disease). Five year survival was 55\% for non-familial and $51 \%$ for familial patients (NS). The main predictor of mortality was an LVEF of $<30 \%$. Familial status did not predict mortality. There was no significant difference in follow up LVEF values between the groups.

Conclusions: Five year survival is not significantly different in the familial and non-familial forms of dilated cardiomyopathy.
\end{abstract}

See end of article for authors' affiliations

.....................

Correspondence to: Dr Virginia V Michels, Department of Medical Genetics, Mayo Clinic, 2001 St SW, Rochester, MN 55905, USA;

michels.virginia@mayo.edu

Accepted

7 February 2003
$\mathrm{D}$ ilated cardiomyopathy is a primary myocardial disorder characterised by dilatation and impaired function of one or both ventricles. ${ }^{1}$ Over $20 \%$ of cases of idiopathic dilated cardiomyopathy are familial. ${ }^{2-4}$ The majority of familial and non-familial cases are of unknown cause. Although several gene defects that cause dilated cardiomyopathy have been identified, these account for only a small proportion of cases. Defects in genes encoding cardiac actin, ${ }^{5}$ cardiac $\beta$-myosin heavy chain, troponin $\mathrm{T}^{6}{ }^{6}$ and $\alpha$-tropomyosin ${ }^{7}$ cause some cases of dilated cardiomyopathy without other organ system disease. Mutations in laminA/C cause dilated cardiomyopathy with prominent conduction system involvement, ${ }^{8}$ while defects in $\delta$-sarcoglycan, ${ }^{9}$ dystrophin, desmin, tafazzin, and the mitochondrial respiratory chain are usually associated with skeletal muscle or other organ involvement in addition to dilated cardiomyopathy. ${ }^{6}$ With the exception of dystrophin, clinical molecular analysis for these gene defects is not available or available on only a very limited basis. Additional chromosomal loci have been linked to dilated cardiomyopathy, for which specific genes have not been identified..$^{10}$ Toxins and infections have been proven to account for a small number of cases. However, the cause of most cases remains unknown.

In most reported series of dilated cardiomyopathy that describe outcome, cases are not separated into familial and non-familial categories. ${ }^{11}$ In part, this is because determining whether or not a patient has a familial disease is often difficult. We and others have shown that classification into familial and non-familial cases requires echocardiographic investigation of relatives. ${ }^{12}$ Asymptomatic relatives may have disease, while relatives reported to have dilated cardiomyopathy by the patient may actually have ischaemic cardiomyopathy or other types of heart disease. Thus it remains unknown whether or not the progression of disease differs in patients with familial and non-familial cardiomyopathy. Anecdotal data have been cited suggesting that some patients with familial disease follow a more rapid course, ${ }^{13}$ whereas in one study the family history did not seem to influence prognosis. ${ }^{14}$ However, in the latter study, families were not specifically investigated to determine whether or not the disease was familial.

We describe a follow up study of survival in a series of patients with dilated cardiomyopathy identified between 1987 and 1992 who were categorised as having familial or non-familial disease based on echocardiographic investigations of relatives. ${ }^{1}$ Although follow up studies of familial dilated cardiomyopathy have been reported, those studies focused on a small number of highly selected families. ${ }^{15}$ Our study is unique because the follow up data relate to cases in whom the diagnosis of familial or non-familial disease was established by echocardiographic studies in first degree relatives.

\section{METHODS}

A retrospective follow up study was conducted of all index patients and their relatives who had been enrolled in studies of dilated cardiomyopathy from 1987 to 1992. This series included 101 patients with the disease who had presented to the Mayo Clinic for cardiology care, without regard to their family history. This unselected series was based on all the patients with idiopathic dilated cardiomyopathy who were evaluated at our institution during this period, without regard to family history, disease severity, or any other factors. All patients had been symptomatic, none having been diagnosed on the basis of screening. As described previously, ${ }^{1}$ these patients had been ascertained by inviting a sequential series of patients to participate in a prospective family study.

In the original study, all first degree relatives had been invited for evaluation, regardless of family history or whether the relative had, or was suspected to have had, heart disease. In the original study families, there were 592 living first degree relatives, of whom $521(88 \%)$ agreed to participate. Evaluation of all participating relatives at the time of the original study had included a review of medical history, lifestyle patterns, cardiovascular physical examination, ECG, and comprehensive cross sectional and M mode echocardiography. Criteria for a diagnosis of dilated cardiomyopathy in 


\begin{tabular}{llll}
\hline $\begin{array}{l}\text { Table } 1 \text { Comparison of familial and non-familial index patients with dilated } \\
\text { cardiomyopathy }\end{array}$ & \multicolumn{3}{l}{} \\
\hline & Familial & Non-familial & p Value \\
\hline Number of patients & 30 & 71 & \\
Age at study entry (years) (mean (SD)) & $51.2(13.1)$ & $48.8(17.6)$ & 0.434 \\
Range & $(18.4$ to 72.5$)$ & $(0.5$ to 75$)$ & \\
Length of follow up (years) (mean (SD)) & $6.1(4.7)$ & $5.9(4.2)$ & 0.864 \\
Range & $(0.1$ to 13$)$ & 10 to 12.6$)$ & \\
Age at follow up (years) (mean (SD)) & $58.7(14.2)$ & $56.4(17.4)$ & 0.495 \\
Range & $(19.1$ to 79.9$)$ & $(24.7$ to 80.2$)$ & \\
Number deceased & 12 & 29 & \\
Number transplanted & 5 & 18 & \\
Age at death or transplant (years) (mean (SD)) & $57.4(15.4)$ & $59.0(14.2)$ & \\
$\quad$ Range & $(19.1$ to 79.9) & $(24.7$ to 80.2) & \\
Five year survival (\%) & 51.5 & 55.7 & 0.561 \\
\hline
\end{tabular}

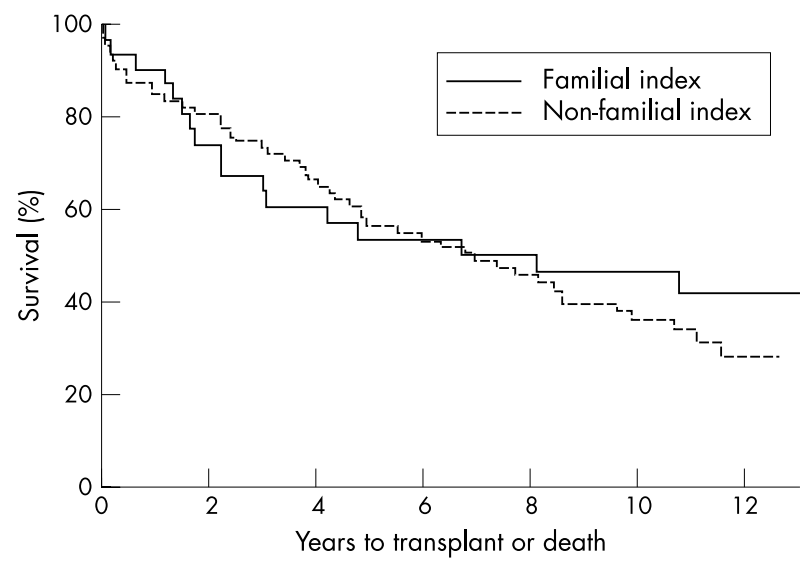

Figure 1 Kaplan-Meier survival curve for index patients, familial and non-familial.

patients and relatives were a left ventricular ejection fraction (LVEF) of $<50 \%$ and left ventricular size above the 95th centile for age and body size. For subjects aged over age 40 years, a diagnosis of dilated cardiomyopathy had been made only if the patient had coronary angiography showing no lesion that caused $\geqslant 50 \%$ vessel obstruction. Patients were excluded if they had a previous myocardial infarct, diabetes mellitus, or other disease that could have resulted in a dilated heart with a decreased ejection fraction.

As described in our previous study, there was no significant difference between the familial and the non-familial cases at the onset of the longitudinal study period with regard to LVEF, left ventricular size in systole or diastole, exercise pattern, number of viable pregnancies, cigarette smoking, number of hospital admissions for viral illness, asthma, or the use of alcohol, caffeine, or drugs for allergies or asthma. Details are as previously described. ${ }^{1}$

For the purpose of this current study, an index patient was classified as having "familial dilated cardiomyopathy" if one or more first degree relatives fulfilled criteria for a diagnosis of dilated cardiomyopathy before or at the time of the current study.

The current follow up study was undertaken by reviewing the Mayo Clinic records of index patients and participating relatives. Permission to review medical records for research purposes was obtained, and the study was approved by the Mayo institutional review board. In addition, the following information was obtained by mail about medical care received elsewhere:

- whether a diagnosis of dilated cardiomyopathy or other type of heart disease had been made after the original study, and if so how the diagnosis was made
- whether an echocardiogram, heart catheterisation, coronary angiogram, cardiac nuclear scan, or heart transplantation had been done since the original study

- the date of death and death certificates of deceased patients.

Permission forms were requested for signatures for release of outside medical information to verify responses, and to obtain details of information, such as LVEF. No data were used without receipt and verification of these records. Medical records, questionnaires, and death certificates were reviewed and abstracted by the principal investigator.

\section{Statistics}

Age, baseline LVEF, and changes in LVEF were compared between the two groups using the two sample $t$ test. The $\chi^{2}$ test was used to compare categorical variables (such as sex) between groups. Kaplan-Meier curves were used to estimate five year survival probabilities. Survival time was censored at heart transplantation. The Cox proportional hazards model was used to calculate the influence of the main baseline characteristics (age, sex, baseline LVEF, and familial status) on survival. Age was expressed as a relative hazard for each 10 year increase. A stepwise model selection method was used to build the multivariate Cox models.

\section{RESULTS}

Of the 101 index patients, 51 were male (mean age at study entry 47.3 years, range $0.5-75$ years), and 50 were female (mean age at study entry 51.6 years, range $13.7-72.5$ years). These ages were not significantly different $(p=0.245)$. Based on the original study, familial dilated cardiomyopathy was present in 20 of these 101 patients. There were no significant age or sex differences between index patients with familial or non-familial dilated cardiomyopathy $(p=0.623$ and $\mathrm{p}=0.961$, respectively). Ninety nine per cent of the families were white, and 1\% were of African American ancestry. Analyses were performed for all subjects-that is, index patients and relatives-who were diagnosed as having dilated cardiomyopathy at the time of the original study. Analysis of unaffected relatives is the subject of a separate report. ${ }^{16}$

\section{Follow up, index patients}

The mean duration of follow up for the 101 index patients was 6.0 years (range 0 days to 12.9 years). For familial cases, mean duration of follow up was 6.1 years, and for non-familial cases, 5.9 years (table 1 ). Two non-familial index patients were lost to follow up.

At the time of the original study, $20 \%$ of index patients had familial disease. After the current study, the proportion of confirmed familial cases increased to $30 \%$. In reporting results 


\begin{tabular}{|c|c|c|c|c|}
\hline Name of model variable & $\begin{array}{l}\text { Number of } \\
\text { patients }\end{array}$ & $\begin{array}{l}\text { Hazard } \\
\text { ratio }\end{array}$ & $95 \% \mathrm{Cl}$ & $\mathrm{p}$ Value \\
\hline Age: per 10 year increase & 101 & 1.3 & (1.1 to 1.5$)$ & 0.009 \\
\hline Male & 101 & 1.2 & $(0.8$ to 2.0$)$ & 0.403 \\
\hline Ejection fraction $\leqslant 30 \%$ & 100 & 4.4 & (2.1 to 9.4 ) & $<0.001$ \\
\hline Familial DCM & 101 & 0.8 & $(0.5$ to 1.5$)$ & 0.562 \\
\hline
\end{tabular}

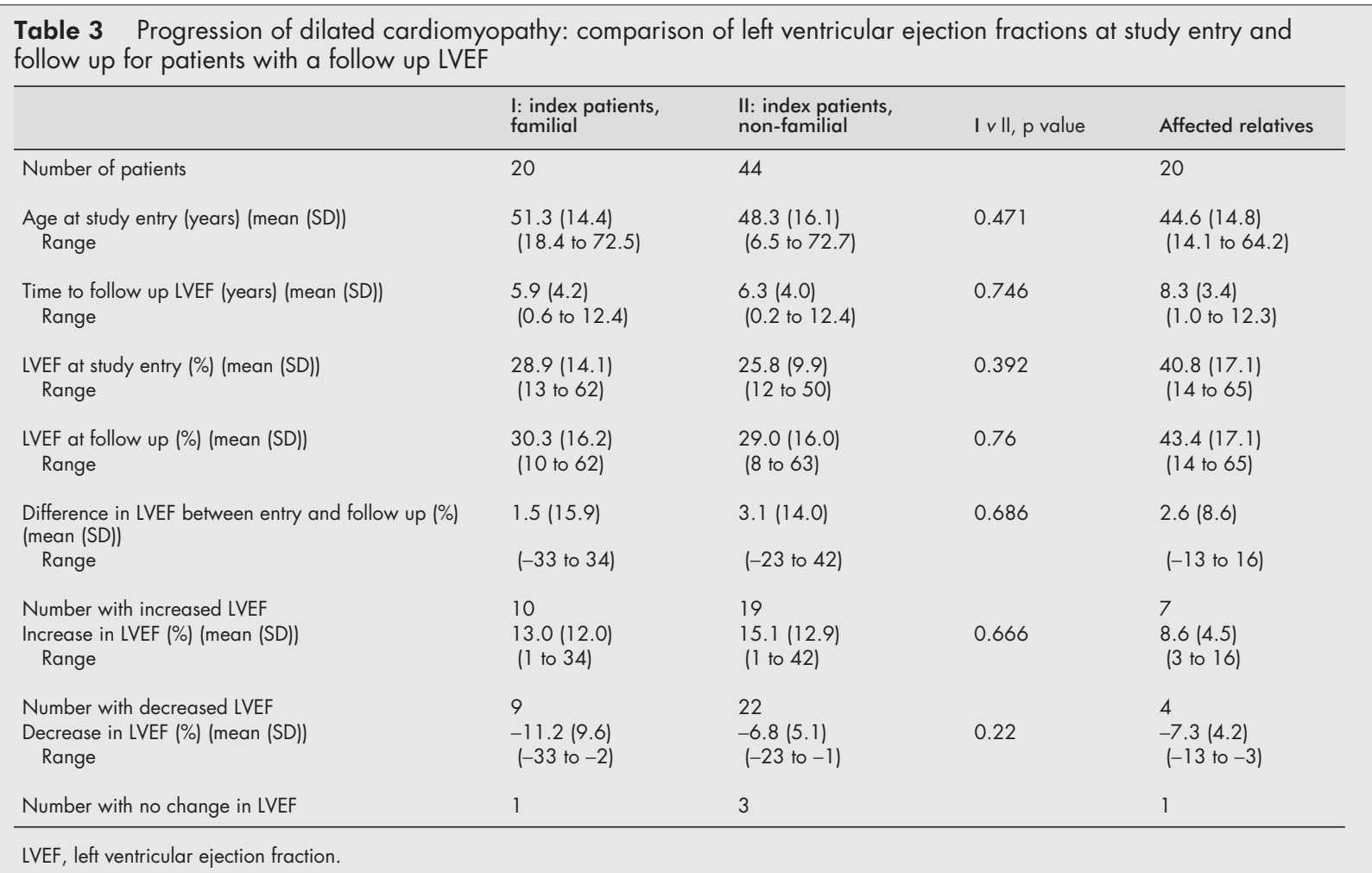

of the present study, familial cases in the series refer to the total number of 30 familial cases ( 16 male and 14 female).

\section{Survival, index patients}

Of the 101 index patients, 41 died and 23 had heart transplantation. The five year survival rates were not significantly different for familial and non-familial index patients $(51.5 \% \vee 55.7 \%, \mathrm{p}=0.561)$ (table $\mathrm{l}$ and fig $\mathrm{l}$ ). The influence of age, sex, LVEF at study entry, and familial status were all examined univariately in a Cox model (table 2). This shows that an LVEF of less than $30 \%$ and older age correlated with decreased survival. After adjusting for LVEF, none of the variables was significant.

\section{LVEF, index patients}

Echocardiograms were done after the original study in 64 of the 101 index patients. For 64 patients (coincidentally the same number of index patients who died, but not necessarily the same patients) the follow up echocardiograms were done on average 6.2 years (range $0.2-12.4$ years) after the initial study (table 3 ). The initial mean LVEF was $26.7 \%$ and the mean (SD) follow up LVEF was 29.3 (16)\%. However, means obscure the fact that some patients improved while others deteriorated. The LVEF improved an average of 14.4 percentage points in 29 patients, declined an average of 8 percentage points in 31 patients, and remained unchanged in four patients. There was no statistical difference in the number of patients whose LVEF increased or declined between the familial and non-familial cases.

\section{Follow up, affected relatives}

During the original study, 33 relatives were identified who had idiopathic dilated cardiomyopathy. Follow up data were obtained on 20 affected relatives. Eleven of these 20 (55\%) had been asymptomatic, with a mean LVEF of $45 \%$. Changes in LVEF for affected relatives with follow up LVEF values are shown in table 3.

\section{DISCUSSION}

Most data on the frequency of familial dilated cardiomyopathy among patients with dilated cardiomyopathy are based on cross sectional studies of families rather than on longitudinal studies. In our original cohort of patients who had been unselected with regard to family history, $20 \%$ had been found to have familial disease after investigation of relatives that had included echocardiographic examination. ${ }^{1}$ There were no characteristics that distinguished familial from non-familial patients-for example, light and electron microscopy ${ }^{17}$ or serum cardiac autoantibodies. ${ }^{18}$ In our follow up study, the frequency of index cases with familial dilated cardiomyopathy increased to $30 \%$ as additional relatives developed the disease. 
Furthermore, family history by patient report neither proved nor disproved familial disease. Some who reported familial involvement had relatives with other forms of heart disease, whereas others with an apparently negative family history had affected relatives on echocardiographic investigation. In our original series, $18 \%$ of index cases would have been misclassified on the basis of family history report. ${ }^{19}$ Because of the difficulty in determining familial status in large series of patients with dilated cardiomyopathy, it is unknown whether the course and prognosis of the disease differs between familial and non-familial cases. It has been suggested that patients with familial disease have a worse prognosis than those with non-familial disease. In a study of 240 Hungarian patients with dilated cardiomyopathy, of whom 32 were familial and 209 were sporadic, the six year survival was $6 \%$ in the familial group and $23 \%$ in the sporadic group. ${ }^{20}$ However, patients in that series were detected by population screening using chest $x$ rays to measure cardiothoracic ratios. In addition, their patients were significantly younger (31.8 years in familial and 39.6 years in non-familial cases) than our index patients. It is not clear if the relatives of index cases in the Hungarian series had echocardiography to look for affected members or if screening included relatives of only those cases suspected of having familial disease. If relatives were highly selected, their sample of familial cases may have been enriched by those with more severe disease-causing genetic factors. The authors concluded that the rapid progression of familial dilated cardiomyopathy should be taken into consideration when planning heart transplantation.

Such a recommendation would be important, because prognosis for patients with dilated cardiomyopathy has been difficult to predict. The five year mortality in reported series of patients with ischaemic and non-ischaemic dilated cardiomyopathy ranges from $75 \%$ to $43 \%$ in those with heart failure. ${ }^{2122}$ For severe congestive heart failure (class III/IV), a one year mortality of $31 \%$ has been reported in patients with dilated cardiomyopathy. ${ }^{23}$ Multiple factors may account for the different survival among series, such as the method of ascertainment, the age of the patients, aetiological heterogeneity, and medical treatment. ${ }^{24}$ In one study that categorised dilated cardiomyopathy into ischaemic and non-ischaemic causes and subdivided non-ischaemic cases into idiopathic and other categories, significant differences in survival were found among the groups, with an overall survival at 4.4 years of $69.9 \%$. However, patients with familial dilated cardiomyopathy were not assessed separately. ${ }^{25}$

Many indices have been reported to have prognostic value for dilated cardiomyopathy, but the predictive value differs with the severity of heart failure and among categories of patients-for example, in those with ischaemic as opposed to idiopathic dilated cardiomyopathy. In one study, ${ }^{23}$ LVEF was a prognostic indicator in mild to moderate congestive heart failure, but not in severe congestive heart failure. However, no indices predict the risk of cardiac death or the need for heart transplantation for all categories of patients or for individual patients. ${ }^{22}{ }^{26}$ Furthermore, with one exception none of the studies that addressed prognosis for dilated cardiomyopathy separately categorised familial and non-familial patients. Heart rate variability did not differ in patients with familial and non-familial disease. ${ }^{27}$ Therefore, it is important to identify patient populations with different outcomes so that prognostic variables can be assessed and applied appropriately.

Our series of patients with idiopathic dilated cardiomyopathy, who were analysed separately for mortality and changes in LVEF, are unique in assessing familial and non-familial disease. Longitudinal follow up over a mean of over five years allowed a more complete assignment of cases into familial and non-familial categories compared with assignment at a single time point. Not unexpectedly, the frequency of familial cases increased from $20 \%$ to $30 \%$, based on additional relatives who had developed the disease. A limitation of any study of this type is that additional patients may later prove to have familial disease. However, this problem is insurmountable until all genetic aetiologies of this condition are discovered and can be assessed in large groups of patients. Our data-where a group of patients with confirmed familial disease identified by echocardiographic investigation of relatives is compared with a group with apparently non-familial disease-are unique and provide the best comparison data currently available. Another limitation is that we did not analyse pharmacological treatment, but made the assumption that the patients, who were all referred to one centre for treatment, received optimal therapy.

In our series of index patients, the five year survival rate was $54.4 \%$ - similar to the mortality reported in other series of patients with dilated cardiomyopathy. There was no significant difference in survival between familial $(51.5 \%)$ and nonfamilial patients $(55.7 \%)$. The reason for the difference in results between our study and the Hungarian study is unknown. Within subtypes of familial dilated cardiomyopathy categorised by inheritance pattern, a worse prognosis has been suggested for autosomal recessive cases compared with autosomal dominant cases. ${ }^{12}$ Because our patients generally had inheritance patterns compatible with the most common (autosomal dominant) inheritance, we cannot address the prognosis for rare autosomal recessive subtypes.

\section{Conclusions}

We conclude that there is no significant difference in the clinical course of patients with familial and non-familial dilated cardiomyopathy. Familial status should not be the main consideration in timing heart transplantation in most cases. We cannot exclude the possibility that there may be subsets of familial cases with more severe disease who present at a younger age than our patients, or who have X linked or autosomal recessive inheritance with a worse prognosis. The subjects in our study with familial dilated cardiomyopathy showed a wide variability in disease course, even within the same family (data not shown). This is supported by our earlier reports of the wide range of age of onset in our series of patients, ${ }^{128}$ and by a follow up study of four families with this disease selected for the large numbers of affected relatives, in whom the age of onset and the clinical course were highly variable in the same family. ${ }^{15}$

\section{ACKNOWLEDGEMENTS}

This work was supported by the American Heart Association. A special thank you to Lisa McGovern and Lori Adler for their organisational and secretarial skills.

\section{Authors' affiliations}

V V Michels, Department of Medical Genetics, Mayo Clinic/Foundation, Rochester, Minnesota, USA

D J Driscoll, *T M Olson, Pediatric and Adolescent Medicine, Mayo Clinic/Foundation

F A Miller, Internal Medicine, Mayo Clinic/Foundation

E J Atkinson, C L Olswold, D J Schaid, Health Sciences Research, Mayo Clinic/Foundation

*Also Internal Medicine, Mayo Clinic Foundation

\section{REFERENCES}

1 Johnson RA, Palacios I. Dilated cardiomyopathies of the adult (first of two parts). N Engl J Med 1982;307:1051-8.

2 Michels VV, Moll PP, Miller FA, et al. The frequency of familial dilated cardiomyopathy in a series of patients with idiopathic dilated cardiomyopathy. N Engl J Med 1992;326:77-82.

3 Grunig $\mathbf{E}$, Tasman JA, Kucherer $\mathrm{H}$, et al. Frequency and phenotypes of familial dilated cardiomyopathy. J Am Coll Cardiol 1998;31:186-94.

4 Keeling PJ, Gang Y, Smith G, et al. Familial dilated cardiomyopathy in the United Kingdom. BM 1995;73:417-21.

5 Olson TM, Michels VV, Thibodeau SN, et al. Actin mutations in dilated cardiomyopathy, a heritable form of heart failure. Science 1998;280:750-2. 
6 Kamisago M, Sharma SD, DePalma SR, et al. Mutations in sarcomere protein genes as a cause of dilated cardiomyopathy. N Engl J Med 2000;343: 1688-96

7 Olson TM, Kishimoto NY, Whitby FG, et al. Mutations that alter the surface charge of alpha-tropomyosin are associated with dilated cardiomyopathy. J Mol Cell Cardiol 2001;33:723-32.

8 Fatkin D, MacRae C, Sasaki T, et al. Missense mutations in the rod domain of the lamin a/c gene as causes of dilated cardiomyopathy and conduction-system disease. N Engl J Med 1999;341:1715-24.

9 Tsubata $S$, Bowles KR, Vatta M et al. Mutations in the human delta-sarcoglycan gene in familial and sporadic dilated cardiomyopathy. J Clin Invest 2000; 106:655-62

10 Schönberger J, Seidman CE. Many roads lead to a broken heart: the genetics of dilated cardiomyopathy. Am J Hum Genet 2001;69:249-60.

11 Bahler RC. Assessment of prognosis in idiopathic dilated cardiomyopathy. Chest 2002;121:1016-19.

12 Mestroni L, Rocco C, Gregori D, et al. Familial dilated cardiomyopathy: evidence for genetic and phenotypic heterogeneity. Heart muscle disease study group. J Am Coll Cardiol 1999:34:181-90.

13 Valantine HA, Hunt SA, Fowler MB, et al. Frequency of familial nature of dilated cardiomyopathy and usefulness of cardiac transplantation in this subset. Am J Cardiol 1989;63:959-63.

14 Diaz RA, Obasohan A, Oakley CM. Prediction of outcome in dilated cardiomyopathy. Br Heart J 1987;58:393-9.

15 Crispell KA, Wray A, Ni H, et al. Clinical profiles of four large pedigrees with familial dilated cardiomyopathy. J Am Coll Cardiol 1999;34:837-47.

16 Michels VV, Olso TM, Miller FM, et al. Frequency of development of idiopathic dilated cardiomyopathy among relatives of patients with idiopathic dilated cardiomyopathy Am J Cardiol (in press).

17 Michels VV, Tazelaar HD, Driscoll DJ, et al. Histopathology of familia versus nonfamilial dilated cardiomyopathy. Cardiovasc Pathol 1993;2:219-23.
18 Michels VV, Moll PP, Rodeheffer RJ, et al. Circulating heart autoantibodies in familial as compared with nonfamilial idiopathic dilated cardiomyopathy. Mayo Clin Proc 1994;69:24-7.

19 Goerss JB, Michels VV, Burnett J, et al. Frequency of familial dilated cardiomyopathy. Eur Heart J 1995; 16(suppl 0):2-4.

20 Csanády $M$, Högye $M$, Kallai $A$, et al. Familial dilated cardiomyopathy: a worse prognosis compared with sporadic forms. Br Heart $J$ 1995;74:171-3

21 Abraham WT, Bristow MR. Specialized centers for heart failure management. Circulation 1997;96:2755-7.

22 Lapu-Bula R, Robert A, De Kock M, et al. Risk stratification in patients with dilated cardiomyopathy: contribution of Doppler-derived left ventricular filling. Am J Cardiol 1998;82:779-85.

23 Alla $F$, Briancon S, Juilliere $Y$, et al. Differential clinical prognostic classifications in dilated and ischemic advanced heart failure: the EPICAL study. Am Heart J 2000;139:895-904.

24 Kawai K, Takaoka H, Hata K, et al. Prevalence, predictors, and prognosis of reversal of maladaptive remodeling with intensive medical therapy in idiopathic dilated cardiomyopathy. Am J Cardiol 1999;84:671-6.

25 Felker GM, Thompson RE, Hare JM, et al. Underlying causes and long-term survival in patients with initially unexplained cardiomyopathy. N Engl J Med 2000;342:1077-84

26 Pinamonti B, Zecchin M, Di Lenarda A, et al. Persistence of restrictive left ventricular filling pattern in dilated cardiomyopathy: an ominous prognostic sign. J Am Coll Cardiol 1997;29:604-12

27 Yi G, Goldman JH, Keeling PJ, et al. Heart rate variability in idiopathic dilated cardiomyopathy: relation to disease severity and prognosis. Heart 1997:77:108-14.

28 Schmidt MA, Michels VV, Edwards WD, et al. Familial dilated cardiomyopathy. Am J Med Genet 1988;31:135-43.

\section{IMAGES IN CARDIOLOGY}

\section{Partial anomalous pulmonary venous connection or scimitar syndrome}

S cimitar syndrome is a rare congenital disorder characterised by an anomalous connection of the pulmonary vein with the inferior vena cava. The anomalous vein appears as a "scimitar"-like shadow on a chest $x$ ray and classically runs from the middle of the right lung to the cardiophrenic angle. We present a scimitar syndrome in a 77 year old woman referred for evaluation of recurrent pleuritis.

Cine magnetic resonance imaging (below left: Ao, aorta ascendens; LPA, left pulmonary artery; IVC, inferior vena cava) revealed an abnormal venous drainage of the right lung via one large pulmonary vein which drains at the inferior vena cava-right atrial junction (arrows). The right lung and airways appeared to be normal in size. The abnormal pulmonary venous drainage from the right lung was confirmed on the levophase of the right pulmonary artery angiogram (below right), which showed that the venous return from the right lung was partly directed via one anomalous vein to the inferior

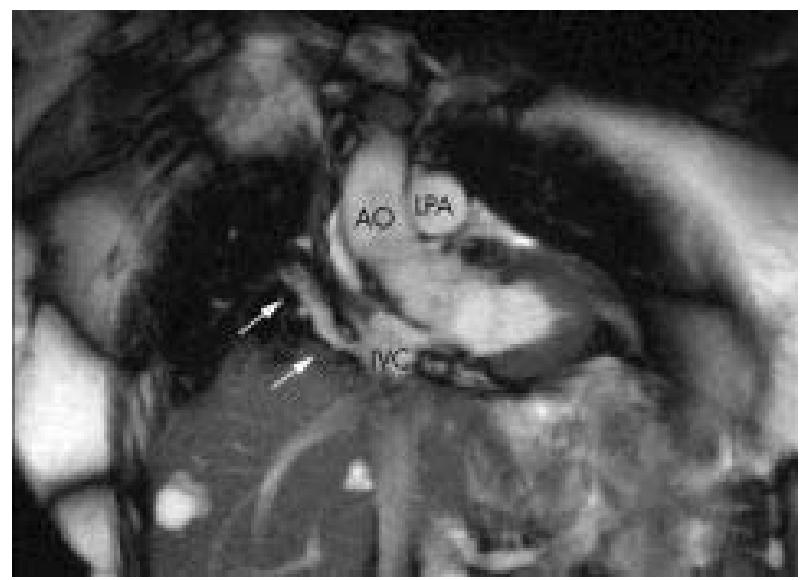

vena cava (arrows). The left pulmonary veins as well as the right upper lobe pulmonary vein connected and drained normally.

Partial anomalous pulmonary venous connection is usually associated with a normal or near normal life span. Many cases do not cause symptoms and are diagnosed incidentally. The natural prognosis is excellent.
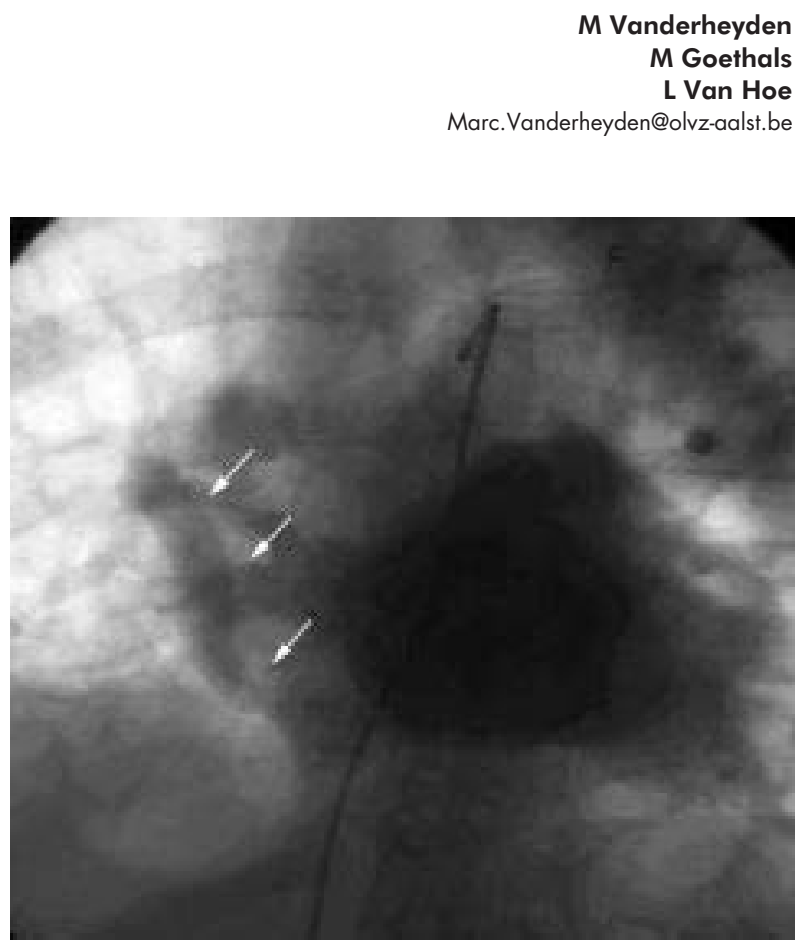